\title{
28 Research Square \\ Short-Term High-Fat Diet Promotes Increased Lysine Crotonylation in Cerebral Cortex
}

\author{
Junling Wang \\ Wenzhou Medical University \\ Jiajia Zhang \\ Wenzhou Medical University \\ Xiaolu Wang \\ Wenzhou Medical University \\ Chaojin Xu ( $\nabla$ doctordoctor79@163.com) \\ Wenzhou Medical College: Wenzhou Medical University https://orcid.org/0000-0003-3927-2321
}

\section{Short Report}

Keywords: Crotonylation, Cerebral cortex, Congo red, High-fat, Lysine, Short-term

Posted Date: September 27th, 2021

DOI: https://doi.org/10.21203/rs.3.rs-878125/v1

License: (9) This work is licensed under a Creative Commons Attribution 4.0 International License. Read Full License 


\section{Abstract}

Protein lysine crotonylation is a newly discovered protein post-translational modification (PTM), which has been associated with cellular metabolism, cell cycle, gene transcription, DNA damage response. However, its potential roles related to human central nervous system diseases remain largely unknown. In the present study, we observed a significant elevated lysine crotonylation in a screening of nine lysine acylations in cortex tissues of HFD-fed mice after short-term overfeeding. On the base of previous reports and molecular weight of proteins, we also speculate that actin, ERK2 or GAPDH and CDK1 might be modified by lysine crotonylation $(\mathrm{KCr})$. Taken together, our findings highlight a potential role of protein lysine crotonylation in HFD-induced brain disorders and as possible therapeutic candidates in the future.

\section{Introduction}

In living organisms and individual cells, post-translational modifications (PTMs) of proteins are crucial for regulation diverse cellular functions, such as DNA replication, transcription, tissue differentiation, apoptosis, inflammation and so on (Berdasco and Esteller, 2010; Lee, 2013). To date, more than 500 discrete types of PTMs across all 20 protein amino acids have been identified (Keenan et al., 2021). In addition to extensive studies of common PTMs, like phosphorylation and acetylation (Acetyllysine, KAc) (Verdin and Ott, 2015), several novel protein acetylation, such as, Lysine malonylation (Malonyllysine, KMal) (Xie et al., 2012), Lysine succinylation (Succinyllysine, KSu) (Xie et al., 2012), Lysine lactylation (Lactyllysine, KLa) (Qin et al., 2019), Lysine propionylation (Propionyllysine, KPr) (Chen et al., 2007), Lysine butyrylation (Butyryllysine, KBu) (Chen et al., 2007), Lysine $\beta$-hydroxybutyrylation ( $\beta$ hydroxybutyryllysine, KHb) (Xie et al., 2016), Lysine glutarylation (Glutaryllysine, KGI) (Tan et al., 2014) and Lysine crotonylation (Crotonyllysine, $\mathrm{KCr}$ ) (Tan et al., 2011) have been discovered in recent years. Moreover, these acylations have been associated with cellular metabolism (Sabari et al., 2017). Among aforementioned lysine acylations, $\mathrm{KCr}$ is involved in the diverse physiopathologic processes of some diseases, such as, neuropsychiatric disorder, acute kidney injury, mouse spermatogenesis, Alzheimer's disease (AD) and cancers (Liu et al., 2017; Sun et al., 2020; Wan et al., 2019; Wang et al., 2019b). Although Lysine crotonylome reveals many studies of histone crotonylation and non-histone crotonylation (Wu et al., 2017), however, whether the levels of protein $\mathrm{KCr}$ could be affected in HFDinduced diseases have not yet been fully addressed.

High-fat diet (HFD), known as the western diet, has been associated with neuroinflammation, apoptosis, necrosis and cognitive deficits in distinct brain regions (Keshk et al., 2020; Pistell et al., 2010). Recent studies indicates that protein acylation modifications associated with HFD and obesity in brain mainly focus on acetylation (Cai et al., 2020; Chen et al., 2015; Gonçalves et al., 2017). On the other hand, while many studies about lysine crotonylation have been reported since 2011, the influence of short-term HFD on protein $\mathrm{KCr}$ in cerebral cortex remains relatively under explored. In this study, our preliminary research shows that the total KCr levels of cerebral cortex among nine types of lysine acylations are significantly increased after 7 and 21 days HFD compared with chow diet. Meanwhile, current results highlight that $\mathrm{KCr}$ modification might become a potential intervention target to fight against HFD-related brain diseases. 


\section{Materials And Methods}

\section{Reagents}

Congo red (C6767) and Coomassie Blue Fast Staining Solution (P0017) were purchased from SigmaAldrich and Beyotime Biotechnology respectively. All antibodies used in this study are listed in the Key Resources Table 1.

\section{Animals, High-fat diet}

C57BL/6J wild type male mice (8-weeks old) were provided by Gem Phar ma tech Co., Ltd (Nanjing, China). Low fat and no sugar Chow diet (TP23100) and High fat diet (HFD, TP23103, 45\% of energy) were provided by Trophic Animal Feed High-tech Co., Ltd (Nantong, China). After 7 days adaptation, mice were randomly divided into three groups ( $\mathrm{n}=3$ mice/group). Chow diet groups were served as controls. Other two groups were fed with HFD for 7 days and 21days respectively. Mice were subsequently humanely sacrificed under sodium pentobarbital anesthesia. Cerebral cortex was collected and homogenized in RIPA lysis buffer (P0013B). The homogenized tissues were centrifuged at 13,000 rpm for $30 \mathrm{~min}$ and the supernatants were kept for later steps. Mouse studies were reviewed and approved by the Committee Guide of Wenzhou Medical University (ethical number 2019-75; Wenzhou, China.). All mice were maintained on a 12 hours light-dark cycle and temperature $24^{\circ} \mathrm{C}-25^{\circ} \mathrm{C}$ with free access to water and food.

\section{Western blot analysis, Coomassie Blue and Congo red Staining}

Western blot, Coomassie blue and Congo red analysis were carried out as described by us (Wang et al.,

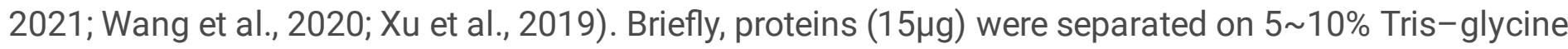
SDS-PAGE gels and transferred onto nitrocellulose membranes. The membranes were then used to perform Congo red staining. Meanwhile, the gels were stained by Coomassie blue. In addition, membranes were probed with primary acyllysine-specific antibodies directed against KMal, KSu, KLa, KPr, $\mathrm{KBu}, \mathrm{KHb}, \mathrm{KGI}, \mathrm{KAc}$ and $\mathrm{KCr}$. Following 3 washes of 10 min each with TBST, a HRP conjugated secondary antibody was used in immunoassays, and then membranes were automated imaged. Subsequently, the membranes were stripped with Beyotime Stripping Buffer (P0025). Then anti-vinculin antibody were run on the Nitrocellulose western blot.

\section{Statistical analysis}

All data were reported as Mean \pm SD (standard deviation). All statistical analyses were performed using GraphPad software (GraphPad Prism version 8.00, San Diego, CA). Differences between two groups were tested by unpaired two-tailed Student's t-test. The significance level were set when ${ }^{*} P<0.05$.

\section{Results}




\section{Short-term HFD feeding increased crotonyllysine levels in mouse cerebral cortex}

To investigate the changes of protein lysine acylations in brain after 21 days short-term HFD, nine types of lysine acylations were detected by western blotting in mouse cerebral cortex lysates. We routinely loaded $15 \mu \mathrm{g}$ total protein onto each line in chow diet and HFD groups, then equal loading was verified by Congo red Nitrocellulose staining and Coomassie blue gels staining (Fig. 1A, B). Subsequently, we observed no changes of $\mathrm{KMal}, \mathrm{KSu}, \mathrm{KLa}, \mathrm{KPr}, \mathrm{KBu}, \mathrm{KAc}, \mathrm{KHb}$ and $\mathrm{KGI}$ in HFD groups compared to chow diet (Fig. 1C-J). Furthermore, although KLa in one group of HFD decreased remarkably, but no significant changes were observed in cerebral cortex of HFD compared to chow diet mice (Fig. 1F). Together, our results suggest that 21 days HFD does not regulate the aforementioned eight modifications of lysine acylations in mouse cerebral cortex. However, we found occasionally that significant elevation of $\mathrm{KCr}$ was observed in HFD groups after 21 days overfeeding compared to chow diet $(P<0.01$, Fig. $1 \mathrm{~K}-\mathrm{L})$. In addition, to explore whether the increase of $\mathrm{KCr}$ levels also could occur at day 7 after HFD, we detected protein $\mathrm{KCr}$ and observed significant elevation of $\mathrm{KCr}$ expressions in HFD groups compared to chow diet $(\mathrm{P}<0.05$, Fig. $1 \mathrm{M}-\mathrm{N})$. In conclusion, our research indicate that $\mathrm{KCr}$ elevation might play major roles in multiple biological processes in mouse cerebral cortex exposed to short-term HFD.

\section{Discussion}

In the current study, we analyzed the nine types of acylation patterns in chow diet and HFD mice after short-term overfeeding. We observed an elevation of protein $\mathrm{KCr}$ levels in cerebral cortex tissues of chow diet and HFD animal models. Yet other eight acylation categories, such as, KMal, KSu, KLa, KPr, KBu, KAc, $\mathrm{KHb}$ and $\mathrm{KGI}$ did not demonstrate significantly changes in HFD groups compare with chow diet. Finally, we hypothesize that brain cortex-specific elevation of $\mathrm{KCr}$ might be common in short-term HFD.

Regular mechanisms of $\mathrm{KCr}$ modification are involved in Lysine crotonyltranferase (KCTs, writer), decrotonylase (KDCRs, eraser) and reader. Currently, KCTs are grouped into three major families: P300/CBP (p300/CREB-binding protein), MYST (Moz, Ybf2, Sas2, Tip60), and GNAT (GCN5-related Nacetyltransferase) in metazoans (Qin et al., 2019). In addition, KDCRs can be classfied into two major categories: HDAC I and HDAC III (Qin et al., 2019). Crotonate can not only be generated by colon microbial fermentation, but also is the short-chain fatty acid (SCFA) precursor of crotonyl-CoA (Stilling et al., 2016). Convertion of SCFA crotonate into crotonyl-CoA is mediated by Acyl-CoA synthetase short chain family member 2 (ACSS2) or other metabolic pathways, such as fatty acid $\beta$-oxidation pathway and the lysine degradation pathway (Sabari et al., 2015) (Lin et al., 2012). Currently, the data featured in the literatures show that $\mathrm{KCr}$ modifications are involved in the aberrant neuroanatomical structures of BTBR mice (Wang et al., 2019a), depressive disorder of male mice (Liu et al., 2019) in the central nervous system. At the same time, we also observed two increased $\mathrm{KCr}$ modifications bands adjacent to 40 and 35kDa at day 7 and 21 after HFD. Because of no available $\mathrm{KCr}$ antibody for immunoprecipitation, we cannot confirm the exact proteins modified by $\mathrm{KCr}$ in HFD groups. However, we speculated that $\mathrm{KCr}$ proteins 
might be actin, ERK2 or GAPDH and CDK1, according to molecular weight of these proteins and a report from high-resolution liquid chromatography-tandem MS (LC-MS/MS) (Xu et al., 2017).

In summary, elevated $\mathrm{KCr}$ modifications in cerebral cortex may exert direct influence over regulating metabolism and other unknown functions in short-term HFD. Future work in this field should focus on the identification of specific proteins related to $\mathrm{KCr}$ modifications, and evaluate their roles whether these are protective or pathogenic responses in HFD-induced brain diseases.

\section{Abbreviations}

Malonyllysine: KMal, Succinyllysine: KSu, Lactyllysine: KLa, Propionyllysine: KPr, Butyryllysine: KBu, $\beta$ hydroxybutyryllysine: KHb, Glutaryllysine:KGI, Acetyllysine: KAc, Crotonyllysine: KCr, High-fat diet: HFD, Post-translational modifications: PTMs, short-chain fatty acid :SCFA, Acyl-CoA synthetase short chain family member 2 :ACSS2.

\section{Declarations}

\section{Acknowledgements}

The study was supported by the Zhejiang Provincial Natural Science Foundation of China (grant no, LY18H090013).

\section{Availability of data and materials}

All data generated or analyzed during this study are included in this published article.

\section{Authors' contributions}

Wang Jun-Ling, Jia-Jia Zhang, Xiao-Lou Wang and Xu Chao-Jin analyzed the data; Xu Chao-Jin, and Wang Jun-Ling wrote the manuscript.

\section{Ethics approval and consent to participate}

Not applicable.

\section{Patient consent for publication}

Not applicable.

\section{Competing interests}

The authors declare that they have no competing interests.

\section{References}


1. Berdasco M, Esteller M (2010) Aberrant epigenetic landscape in cancer: how cellular identity goes awry. Dev Cell 19:698-711

2. Cai HB, Fan ZZ, Tian T, Li ZC, Zhao CC, Guo WT, Ge ZM (2020) Diabetes-Induced H3K9 Hyperacetylation Promotes Development of Alzheimer's Disease Through CDK5. J Alzheimers Dis 77:75-84

3. Chen Y, Sprung R, Tang Y, Ball H, Sangras B, Kim SC, Falck JR, Peng J, Gu W, Zhao Y (2007) Lysine propionylation and butyrylation are novel post-translational modifications in histones. Mol Cell Proteomics 6:812-819

4. Chen Y, Wu R, Chen HZ, Xiao Q, Wang WJ, He JP, Li XX, Yu XW, Li L, Wang P, Wan XC, Tian XH, Li SJ, Yu X, Wu Q (2015) Enhancement of hypothalamic STAT3 acetylation by nuclear receptor Nur77 dictates leptin sensitivity. Diabetes 64:2069-2081

5. Gonçalves LK, da Silva IRV, Cechinel LR, Frusciante MR, de Mello AS, Elsner VR, Funchal C, Dani C (2017) Maternal consumption of high-fat diet and grape juice modulates global histone $\mathrm{H} 4$ acetylation levels in offspring hippocampus: A preliminary study. Neurosci Lett 661:29-32

6. Keenan EK, Zachman DK, Hirschey MD (2021) Discovering the landscape of protein modifications. Mol Cell 81:1868-1878

7. Keshk WA, Ibrahim MA, Shalaby SM, Zalat ZA, Elseady WS (2020) Redox status, inflammation, necroptosis and inflammasome as indispensable contributors to high fat diet (HFD)-induced neurodegeneration; Effect of N-acetylcysteine (NAC). Arch Biochem Biophys 680:108227

8. Lee S (2013) Post-translational modification of proteins in toxicological research: focus on lysine acylation. Toxicol Res 29:81-86

9. Lin H, Su X, He B (2012) Protein lysine acylation and cysteine succination by intermediates of energy metabolism. ACS Chem Biol 7:947-960

10. Liu S, Yu H, Liu Y, Liu X, Zhang Y, Bu C, Yuan S, Chen Z, Xie G, Li W, Xu B, Yang J, He L, Jin T, Xiong Y, Sun L, Liu X, Han C, Cheng Z, Liang J, Shang Y (2017) Chromodomain Protein CDYL Acts as a Crotonyl-CoA Hydratase to Regulate Histone Crotonylation and Spermatogenesis. Mol Cell 67:853866.e855

11. Liu Y, Li M, Fan M, Song Y, Yu H, Zhi X, Xiao K, Lai S, Zhang J, Jin X, Shang Y, Liang J, Huang Z (2019) Chromodomain Y-like Protein-Mediated Histone Crotonylation Regulates Stress-Induced Depressive Behaviors. Biol Psychiatry 85:635-649

12. Pistell PJ, Morrison CD, Gupta S, Knight AG, Keller JN, Ingram DK, Bruce-Keller AJ (2010) Cognitive impairment following high fat diet consumption is associated with brain inflammation. $J$ Neuroimmunol 219:25-32

13. Qin S, Sun D, Zhang C, Tang Y, Zhou F, Zheng K, Tang R, Zheng Y (2019) Downregulation of sonic hedgehog signaling in the hippocampus leads to neuronal apoptosis in high-fat diet-fed mice. Behav Brain Res 367:91-100

14. Sabari BR, Tang Z, Huang H, Yong-Gonzalez V, Molina H, Kong HE, Dai L, Shimada M, Cross JR, Zhao Y, Roeder RG, Allis CD (2015) Intracellular crotonyl-CoA stimulates transcription through p300- 
catalyzed histone crotonylation. Mol Cell 58:203-215

15. Sabari BR, Zhang D, Allis CD, Zhao Y (2017) Metabolic regulation of gene expression through histone acylations. Nat Rev Mol Cell Biol 18:90-101

16. Stilling RM, van de Wouw M, Clarke G, Stanton C, Dinan TG, Cryan JF (2016) The neuropharmacology of butyrate: The bread and butter of the microbiota-gut-brain. axis? Neurochem Int 99:110-132

17. Sun CF, Xu WF, Zhao QW, Luo S, Chen XA, Li YQ, Mao XM (2020) Crotonylation of key metabolic enzymes regulates carbon catabolite repression in Streptomyces roseosporus. Commun Biol 3:192

18. Tan M, Luo H, Lee S, Jin F, Yang JS, Montellier E, Buchou T, Cheng Z, Rousseaux S, Rajagopal N, Lu Z, Ye Z, Zhu Q, Wysocka J, Ye Y, Khochbin S, Ren B, Zhao Y (2011) Identification of 67 histone marks and histone lysine crotonylation as a new type of histone modification. Cell 146:1016-1028

19. Tan M, Peng C, Anderson KA, Chhoy P, Xie Z, Dai L, Park J, Chen Y, Huang H, Zhang Y, Ro J, Wagner GR, Green MF, Madsen AS, Schmiesing J, Peterson BS, Xu G, Ilkayeva OR, Muehlbauer MJ, Braulke T, Mühlhausen C, Backos DS, Olsen CA, McGuire PJ, Pletcher SD, Lombard DB, Hirschey MD, Zhao Y (2014) Lysine glutarylation is a protein posttranslational modification regulated by SIRT5. Cell Metab 19:605-617

20. Verdin E, Ott M (2015) 50 years of protein acetylation: from gene regulation to epigenetics, metabolism and beyond. Nat Rev Mol Cell Biol 16:258-264

21. Wan J, Liu H, Ming L (2019) Lysine crotonylation is involved in hepatocellular carcinoma progression. Biomed Pharmacother 111:976-982

22. Wang JL, Chen WG, Zhang JJ, Xu CJ (2021) Nogo-A- $\Delta 20 /$ EphA4 interaction antagonizes apoptosis of neural stem cells by integrating p38 and JNK MAPK signaling. J Mol Histol 52:521-537

23. Wang JL, Zhao L, Li MQ, Chen WG, Xu CJ (2020) A sensitive and reversible staining of proteins on blot membranes. Anal Biochem 592:113579

24. Wang M, Chang Q, Yang H, Liu Y, Wang C, Hu F, Wei H, Li R (2019a) Elevated lysine crotonylation and succinylation in the brains of BTBR mice. Int J Dev Neurosci 76:61-64

25. Wang Z, Zhao Y, Xu N, Zhang S, Wang S, Mao Y, Zhu Y, Li B, Jiang Y, Tan Y, Xie W, Yang BB, Zhang Y (2019b) NEAT1 regulates neuroglial cell mediating $A \beta$ clearance via the epigenetic regulation of endocytosis-related genes expression. Cell Mol Life Sci 76:3005-3018

26. Wu Q, Li W, Wang C, Fan P, Cao L, Wu Z, Wang F (2017) Ultradeep Lysine Crotonylome Reveals the Crotonylation Enhancement on Both Histones and Nonhistone Proteins by SAHA Treatment. J Proteome Res 16:3664-3671

27. Xie Z, Dai J, Dai L, Tan M, Cheng Z, Wu Y, Boeke JD, Zhao Y (2012) Lysine succinylation and lysine malonylation in histones. Mol Cell Proteomics 11:100-107

28. Xie Z, Zhang D, Chung D, Tang Z, Huang H, Dai L, Qi S, Li J, Colak G, Chen Y, Xia C, Peng C, Ruan H, Kirkey M, Wang D, Jensen LM, Kwon OK, Lee S, Pletcher SD, Tan M, Lombard DB, White KP, Zhao H, Li J, Roeder RG, Yang X, Zhao Y (2016) Metabolic Regulation of Gene Expression by Histone Lysine $\beta$ Hydroxybutyrylation. Mol Cell 62:194-206 
29. Xu CJ, Wang JL, Jing P, Min L (2019) Tph2 Genetic Ablation Contributes to Senile Plaque Load and Astrogliosis in APP/PS1 Mice. Curr Alzheimer Res 16:219-232

30. Xu W, Wan J, Zhan J, Li X, He H, Shi Z, Zhang H (2017) Global profiling of crotonylation on nonhistone proteins. Cell Res 27:946-949

\section{Tables}

Due to technical limitations, table 1 is only available as a download in the Supplemental Files section.

\section{Figures}



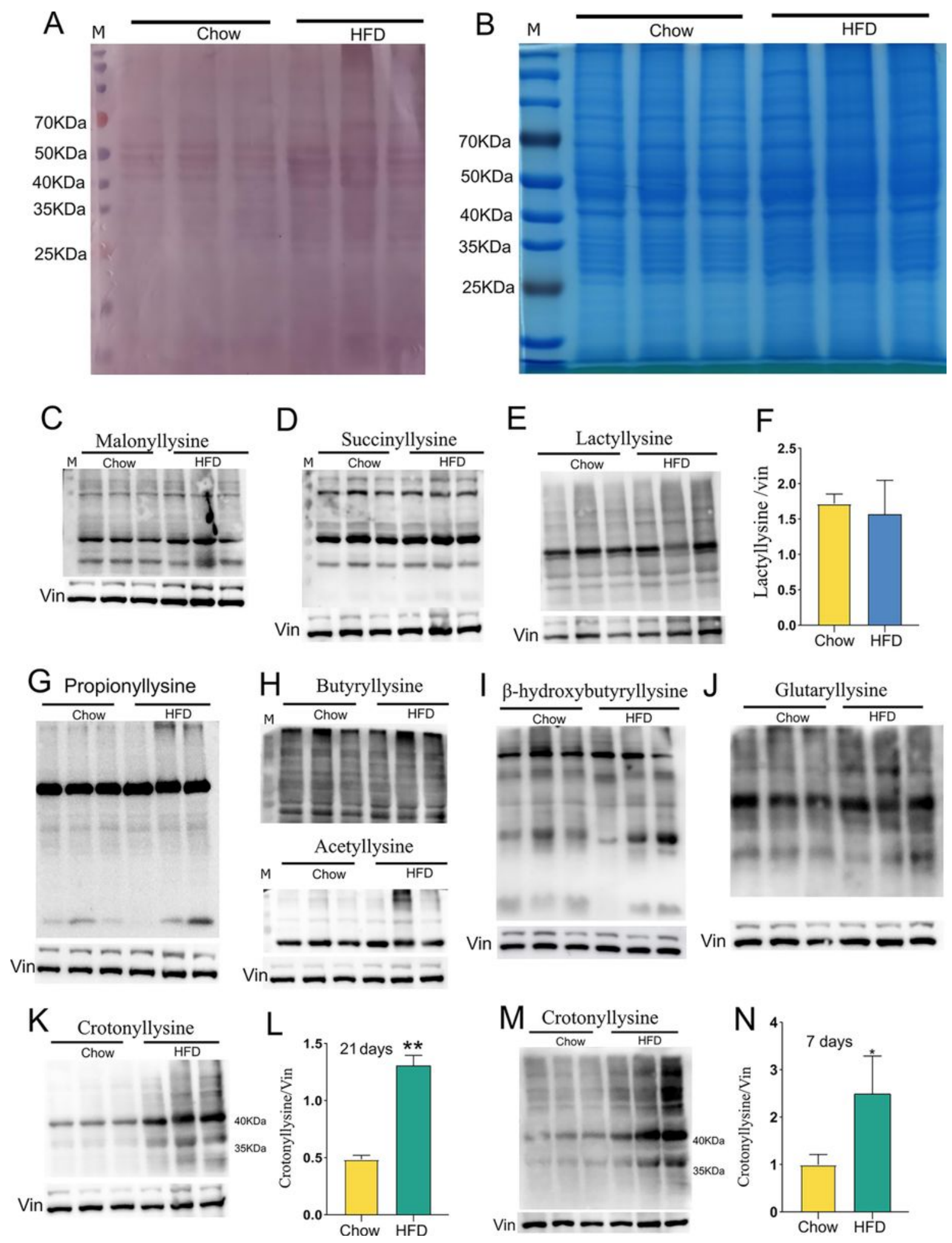

\section{Figure 1}

Nine lysine acylation changes in cerebral cortex under short-term chow diet and HFD feeding. Supernatants were collected from mice brain cortex fed by a chow diet $(n=3$ mice) and HFD for 7 days ( $n=3$ mice) or 21 days ( $n=3$ mice), which were followed by staining and blots. (A) Congo red Staining and (B) Coomassie blue staining serve as a loading control. (C-L) Western blots detecting of cortex protein acetylation with different acyllysine-specific antibodies after 21 days overnutrition (C) KMal, (D) KSu, and 
(E) KLa. (F) Quantification of KLa in (E). (G) KPr, (H) KBu and KAc. (I) KHb, (J) KGI and (K) KCr. (L) Quantification of $\mathrm{KCr}$ in (K). (M) KCr at day 7. (N) Quantification of $\mathrm{KCr}$ in (M). Protein molecular weight markers are indicated on the left-hand side. The levels of acyllysine-specific related proteins were normalized to the levels of vinculin. Values are presented as means $\pm S D$. ${ }^{*} P<0.05$ and ${ }^{* * P}<0.01$ versus chow diet; Two-tailed Student's t test. M: Marker, Chow: Chow diet, HFD: High-fat diet, Vin: vinculin.

\section{Supplementary Files}

This is a list of supplementary files associated with this preprint. Click to download.

- Table.1KeyResources.tif 